\title{
Sedentary Lifestyle
}

National Cancer Institute

\section{Source}

National Cancer Institute. Sedentary Lifestyle. NCI Thesaurus. Code C86589.

A type of lifestyle that lacks physical exercise, characterized by sitting, reading, watching television or using a computer for much of the day without vigorous physical exertion. 